Voix et Images

volxetimages

\title{
Deux rétrospectives. Madeleine Gagnon, Alexis Lefrançois
}

\section{André Brochu}

Volume 33, numéro 1 (97), automne 2007

Michel Marc Bouchard

URI : https://id.erudit.org/iderudit/017536ar

DOI : https://doi.org/10.7202/017536ar

Aller au sommaire du numéro

\section{Éditeur(s)}

Université du Québec à Montréal

\section{ISSN}

0318-9201 (imprimé)

1705-933X (numérique)

Découvrir la revue

Citer ce compte rendu

Brochu, A. (2007). Compte rendu de [Deux rétrospectives. Madeleine Gagnon, Alexis Lefrançois]. Voix et Images, 33(1), 158-163.

https://doi.org/10.7202/017536ar d'utilisation que vous pouvez consulter en ligne.

https://apropos.erudit.org/fr/usagers/politique-dutilisation/ 


\author{
P O É S I E \\ Deux rétrospectives. \\ Madeleine Gagnon, Alexis Lefrançois \\ $+\quad+$
}

ANDRÉ BROCHU

Université de Montréal

Après les rétrospectives de Pierre Nepveu et d'Hélène Dorion ${ }^{1}$, voici celles de Madeleine Gagnon ${ }^{2}$ et d'Alexis Lefrançois ${ }^{3}$, qui présentent toutes deux la caractéristique d'être la reprise, totale ou partielle, de rétrospectives antérieures parues au début des années 1980. Depuis Autographie I' ${ }^{4}$ l'œuvre de Madeleine Gagnon s'est considérablement enrichie, de sorte qu'on peut considérer que l'essentiel est venu après coup; alors que L'œuf à la noix n'ajoute pas de textes nouveaux aux poèmes de Comme tournant la page ${ }^{5}$, l'auteur s'étant surtout illustré depuis, sous la signature d'Ivan Steenhout, qui est son nom véritable, dans la traduction littéraire ${ }^{6}$.

\title{
LE CHANT LOYAL DE MADELEINE GAGNON
}

«[T]u as trouvé l'énigme/tu cherchais le poème» (725), lit-on au terme de la volumineuse édition de l'œuvre poétique de Madeleine Gagnon. Et c'est vrai : le poème n'est jamais le poème. Il doit être autre chose - cri, méditation, effraction de rêve, parfois procès-verbal d'une réalité porteuse de sens... Chez Madeleine Gagnon, il est surtout énigme, c'est-à-dire quelque chose de très simple et d'impénétrable, de quotidien et d'infiniment lointain. Claudel l'avait dit déjà : «Les mots que j'emploie,/Ce sont les mots de tous les jours, et ce ne sont point les mêmes ${ }^{7}$ !» Et Gagnon, qui est une femme de gauche, est d'accord avec le gros homme catholique (voir p. 676), ce qui montre que chez elle, la poétique sait l'emporter sur la politique.

1 On pourra lire un compte rendu sur celle de Pierre Nepveu dans «Fils déchus et autres matières », Voix et Images, vol. XXXI, n² 2, 2006, p. 159-164, et sur Hélène Dorion dans «Les mots-motifs d'Hélène Dorion », Voix et Images, vol. XXXII, n 3, 2007. 2 Madeleine Gagnon, À l'ombre des mots. Poèmes 1964-2006, Montréal, l'Hexagone, coll. «Rétrospectives», 2007, 750 p. 3 Alexis Lefrançois, L'œuf à la noix, poèmes et petites choses, Québec, Éditions Nota bene, coll. «Prose et posée», 2006, 360 p. 4 Madeleine Gagnon, Autographie I. Fictions, Montréal, VLB éditeur, 1982, 300 p. 5 Alexis Lefrançois, Comme tournant la page, 2 tomes, Montréal, Éditions du Noroît, 1984. 6 Signalons toutefois la parution, en 2006, d'un recueil de poèmes intitulé Pages tombées d'un livre (Québec, Le lézard amoureux, 2006), qui a remporté le Prix des terrasses Saint-Sulpice 2007. 7 Paul Claudel, Cinq grandes odes. Suivies d'un processionnal pour saluer le siècle nouveau, Paris, Éditions Gallimard, 1936, p. 108. 
Plus de quarante ans d'écriture, magnifiquement continue et fidèle à la tonalité choisie dès l'origine. Ou presque. Car les deux premiers recueils parus, Pour les femmes et tous les autres ${ }^{8}$ et Poélitique ${ }^{9}$, malmenaient quelque peu l'énigme, au profit des idéologies marxiste et féministe - les deux ensemble, ce qui n'est pas si conforme aux orthodoxies... Madeleine Gagnon les met de côté et choisit pour point de départ Antre, paru en 1978, mais dont l'écriture est, en partie du moins, chronologiquement antérieure. Il s'agit là, écrit-elle dans sa présentation, de son premier vrai recueil, «le nucléus de tout l'œuvre à venir» (12). La métaphore scientifique, "nucléus », qui témoigne chez l'auteure d'une curiosité maintes fois manifestée pour les sciences, en particulier celles de la Terre, suggère un principe inerte et pourtant actif, grain de sable ou fragment, capable d'opérer la cristallisation de minéraux, comme dans la perle. Le lisse, le doux, le translucide sont obtenus à partir d'une rude élaboration. L'« eau» de la perle est la conversion de la pierre, cette pierre qui est un motif fécond de l'œuvre de Gagnon et qui figure dans le titre d'un important recueil (Rêve de pierre ${ }^{10}$ ). On a fréquemment l'impression que la lumière propre au poème est la résultante d'une sourde transmutation, d'un adoucissement énigmatique qui substitue, à la logique âpre des mots de la raison, une tendresse espérante, un orient.

Antre, c'est le ventre. Le ventre de la mère, que la poète pose comme le centre de tout. La mère, l'enfant, le père parfois, sont des figures essentielles du poème. La mère surtout, dont la vérité tragique perce un brouillard de mots pas toujours faciles à déchiffrer. Je cite au complet un poème représentatif :

Tu m'amènes dans cette oasis mauve eau d'amertume dont ils n'ont pas su parler. L'ont imaginée mère stagnante, mère trouble et noire, et troublés de ce manque, de cette perte d'elle, ils ont clamé l'enfance symbiotique ou la mère phallique. Trop nombreuses mères schizogènes ainsi nommées par des fils malades d'inceste, l'ogresse de leur nuit blanche, neige noire, à leur image, dedans, se dévoraient, cette boule des désirs interdits

pendant qu'elles au dehors emmurées dans leurs propres enceintes, assassinées ou violées

ne me demandez plus c'est quoi l'amour

percer le mur du son du sens. (40)

Ce texte vibrant contient plusieurs traits caractéristiques de la poésie de Gagnon, à commencer par l'imprécision volontaire concernant les «acteurs» : «tu», destinataire du discours, mais aussi sujet d'une action qui met le moi en position de témoin, s'agirait-il de l'homme aimé? ; et «ils», ceux qui dressent de la mère un portrait catastrophique et qui pourraient être les tenants de la tradition phallocentrique. Ceux-ci voient leur mère depuis le fond incestueux de leur désir, ils la voient comme

8 Madeleine Gagnon, Pour les femmes et tous les autres, Montréal, L'Aurore, coll. "Lecture en vélocipède», 1974. 9 Id., Poélitique, Montréal, Les Herbes rouges, 1975. 10 Id., Rêve de pierre, Montréal, VLB éditeur, 1999, 171 p. 
être phallique et comme ogresse... On mesure tout de suite ce qui se dissimule, ou plutôt s'affiche à moitié, de savoir dans la méditation poétique de l'auteure. Passionnée, dès le début de sa vie intellectuelle, par les études de symbolique et ce qui les alimente, notamment la psychanalyse; puis conquise par le marxisme et le structuralisme, mais par-dessus tout femme (et féministe), Madeleine Gagnon ne peut assigner un sujet à son inspiration sans que soient convoqués aussitôt des arrièreplans multiples, et tout l'élan de l'émotion esthétique est en même temps une tentative de "percer le mur du son du sens», comme l'affirme la conclusion du texte cité. Investir le ventre, l'antre où germe ce qui donne sens et vie à l'existence, ce qui enfante les destinées humaines, voilà un projet à la fois de l'intelligence et du cœur, et qui suppose aussi une approche modeste des vérités les plus simples.

D'un bout à l'autre de son œuvre poétique, Madeleine Gagnon reprend les mêmes formes discursives: la prose, généralement sans paragraphe et plutôt courte (entre dix et vingt lignes, rarement davantage), et le poème en vers brefs, lui aussi le plus souvent sans division. La poète y parle volontiers d'elle, fait état des circonstances quotidiennes. On a l'impression, assez souvent, de carnets intimes où les circonstances de l'existence sont évoquées avec précision, parfois au mépris de la poésie ("Aujourd'hui vingt-huit novembre, c'est un deuxième été des Indiens, je vais aller me promener il fait trop beau dehors. Le parc DeLorimier n'a jamais été aussi merveilleux.» [220]). Une telle banalité du lyrisme est heureusement rare, mais il ne faut pas non plus la condamner trop vite, car c'est souvent à partir du lieu commun que, chez Gagnon, se dessine le chemin vers le sens à appréhender. On trouve, chez elle, tantôt le très immédiatement accessible et tantôt, fait des mêmes images et des mêmes objets, un hermétisme redoutable, appliqué lui aussi à la quête de l'énigme. On comprend ainsi que l'énigme et le poème ne sont pas synonymes, comme l'affirme la citation qui ouvre le présent texte, mais ils sont tout de même indissociables et mènent l'un à l'autre.

Le point de départ est donc volontiers familier, voire familial. Les enfants occupent leur juste part au sein de l'univers immédiat: "Je les ai bercés au rythme des récits mauves de mes aïeules, en moi.» (33) L'ontogenèse accomplit la phylogenèse, pourrait écrire celle qui affectionne des mots comme "photomicrographie» (156), «métamorphisme» (157), «géoscribe» $(215,219,290)$, « archéographie» (214, 252) et ne dédaigne pas cette préciosité qui nomme les choses par leur nom ("sanguisorbe», «chrysolithe», "péridot», «sardoine», [165]). Oui, la rêverie dirigée vers l'énigme du monde s'accommode volontiers de motifs très précis, tout en restant attentive au sang et au lait (les deux sont souvent associés) matriciels :

\footnotetext{
[...] le temps ne compte plus ni les espaces

on a reçu la terre en héritage

des pères immortels

des mères? qui rêvent et rient les mots

et nous les donnent avec le lait (314).
}

Jusque dans l'un des plus récents recueils est évoqué l'« œil perdu dans les champs anciens de sang et de lait mêlés» (657), où «les mères affolées [...] les pères revenus 
en catastrophe» pleurent les «fils suicidés ». Cette scène d'apocalypse fait contrepoids à la scène heureuse de la citation précédente et montre la sensibilité de l'auteure aux malheurs d'une humanité qui tourne le dos à la sérénité initiale, celle de l'antre originel, de l'éden, souvent évoqué: «la langue bouge/les mots coulent/on crée un huitième jour/on s'invente des destins» (315).

C'est dire que la poésie de Madeleine Gagnon, même quand elle dit je, n'est jamais la fade ritournelle des sentiments individuels. Elle part du moi pour interroger tout de suite ce qu'il y a d'humain dans l'homme et dans la femme - dans la femme d'abord. Il n'y a pas de complaisance pour ce qui distrait des significations les plus élevées. En ce sens, la loyauté est la caractéristique majeure du discours: loyauté à ce qui accomplit l'humain, et le moi par l'humain.

Cela dit, certains textes peuvent sembler plus faibles. L'hermétisme n'est pas toujours au service d'une appropriation du mystère, et quelque naïveté d'allure se manifeste parfois, notamment dans une suite comme «Hé, les choses » (393-420), où une métaphysique du rapport entre les mots et les choses essaie de faire oublier l'austérité du propos par un ton faussement enjoué. On y retrouve toutefois l'expression de préoccupations majeures, comme l'importance du donné immédiat (les choses) et de la musique qui accomplit la chance du monde, en accord avec la poésie.

Pour conclure cette évocation fatalement lacunaire d'un livre gros de toute une vie d'écriture, je ferai quelques réflexions sur le titre ${ }^{11}$. L'énoncé «À l'ombre des mots» reflète bien l'ensemble des recueils réunis. Paradoxalement, il frappe par sa modestie, son absence totale de dimension spectaculaire, tant sur le plan rhétorique que représentatif. "Ombre» et "mots», qui relèvent phonétiquement du même registre sombre, font aussi chiasme par leurs lettres initiales (om - mo). Le langage semble ainsi étendre son empire à tout ce qu'il recouvre et convoque à la cérémonie du sens. L'ombre des mots est cette tendre, souvent passionnée ambiance où se peut entrevoir quelque chose de l'énigme qui justifie l'effort de vivre.

\section{CALCAIRE ET CALVAIRE}

Alexis Lefrançois, né en Belgique, est le plus québécois des poètes venus du vaste monde, comme en fait foi son admirable connaissance des sacres, idiotismes et parlures d'icitte. L'admirable, à vrai dire, c'est ce qu'il fait de nos particularismes qu'il entremêle allègrement à ceux d'ailleurs, par exemple à l'argot français, créant ainsi une langue savoureuse, drôle, cocasse, apte à exprimer des sentiments vifs et saugrenus, d'une étonnante vérité. Cela, dans les Petites choses qui composent plus de la moitié - la dernière - de la rétrospective.

La première partie, intitulée Poèmes, est d'une encre plus orthodoxe, si l'on peut dire. S'y affirme un projet poétique qu'on pourrait, malgré l'usage du vers libre

$$
++
$$

11 Qui devait d'abord être Passerelle, si l'on en juge par la note bibliographique publiée sur le site de l'île. 
et la modernité de l'entreprise, rapprocher de l'esthétique parnassienne, celle de Heredia ou de Leconte de Lisle. Nonobstant la présence de la nature et la chaleur, la spontanéité des rythmes, les thèmes prédominants sont le minéral ("Calcaires » est le titre du premier recueil publié), le blanc qui impose partout sa clarté et sa froide pureté, le cristal, le marbre, la glace, le cygne, la mort et, du côté de l'humain, l'orgueil qui permet d'habiter et de dominer un univers promis à la décomposition:

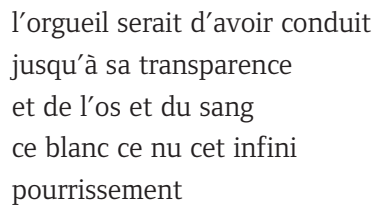

Voilà qui n'est guère conforme à la mentalité du lecteur local, du moins celui d'autrefois, que l'enseignement de l'Église a dressé à rejeter l'orgueil comme la tentation satanique par excellence. Ici, la posture luciférienne mène à un univers réduit au sang et aux os et au refus des "choses", ce qui n'est pas si étranger, en somme, aux perspectives chrétiennes. Mais Lefrançois dit oui là où les effarouchés vertueux disent non. Il est un orgueilleux content. C'est affaire de révolte, face à la société croupie:

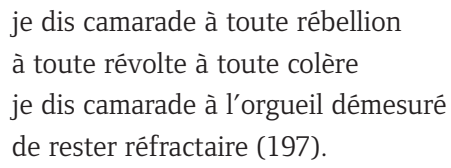

Il y a là un sain anarchisme, sans doute, tel qu'on en trouve peu d'exemples au Québec, et surtout pas dans ces années 1970 où publiait Lefrançois. C'était l'époque du collectivisme militant, la Révolution tranquille s'apprêtait à jeter ses derniers feux pendant qu'un formalisme disert commençait d'enflammer les jeunes esprits. Même à la Nouvelle Barre du Jour, on était loin de l'altier Mallarmé auquel, au-delà du Parnasse, pourrait faire penser Lefrançois.

Aussi, à côté de ses Poèmes ambitieux et un peu guindés, crut-il bon de laisser libre cours en d'autres textes à une extraordinaire fantaisie, sous le titre de Petites choses, lesquelles débutent par un recueil intitulé Petites choses pour la 51. «La 51 », c'est-à-dire l'autobus 51 tel que le désignaient les usagers montréalais en le féminisant (comme tout ce qui est secourable). Et là, l'amateur de stricts calcaires devient un intempérant partisan du mou:

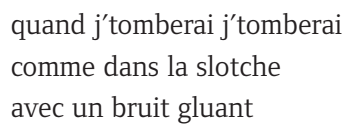




\author{
de vieille affaire molle \\ quand j'tomberai j'tomberai plotch \\ comme une pomme trop mûre \\ ou comme un plat de pâtes \\ j'aurai mon air de poche \\ vidée de ses patates \\ sans os sans âme sans tripes \\ et sans boyaux (167).
}

\begin{abstract}
À l'orgueil bien tempéré de celui qui prétendait réaliser un accord idéal avec le monde, succède l'humilité d'un homme fasciné par ses limites, ses manques, et décidé à les dire sans ménagement en des formules d'une terrible ingénuité. On dirait des comptines rédigées conjointement par Prévert, Mallarmé - et Gérald Godin en sus, pour la touche locale. Ce n'est pas de la «poésie» mais ça parle, ça signifie avec des impétuosités de balles de fusil, et ça dilate la rate, même des imbéciles. C'est du petit texte qui brasse grand, qui retrouve in petto tous les thèmes de la poésie sérieuse et qui permet donc d'y revenir, de trouver tout le grouillement de vie qui se cache sous les apologies du blanc et du glacé.

Voilà donc une rétrospective en deux volets, l'un de cristal et l'autre de gadoue (de slotche, calvaire! de slotche!) et cela brille l'un par l'autre, à contresens et sans limite.
\end{abstract}

